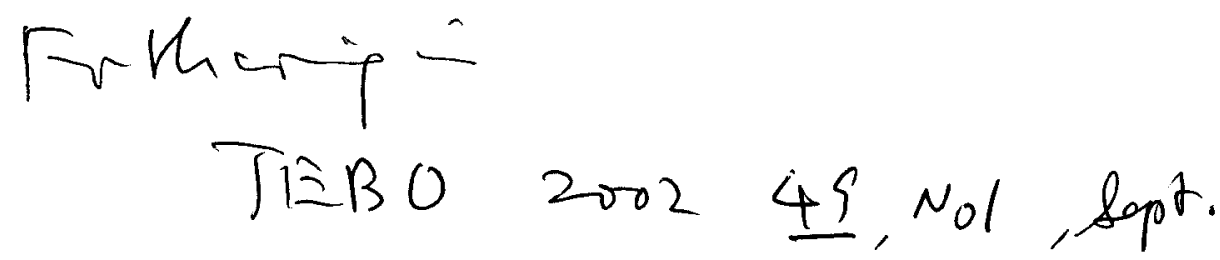

\title{
Open Models of Share Markets with Two Dominant Types of Participants
}

\author{
Masanao Aoki \\ Department of Economics \\ University of California, Los Angeles \\ Fax Number 310-825-9528, e-mail aoki@econ.ucla.edu
}

March 2000; revised July 2001

\begin{abstract}
$^{1}$
This paper examines a share market by using a jump Markov process to model entries, exits and switchings of trading rules by a large number of interacting participants in the market.

The paper examines stationary distributions of clusters of agents by strategies. We concentrate on situations where behavior of market participants are positively correlated. In these cases about 95 percent of the market participants can be shown to belong to two largest subgroups of agents with two trading rules. Contributions of the remaining 5 percent or so of participants are ignored in examining the market behavior as a whole.

Market excess demand and price dynamics are examined in this framework. At the end a possibility for the existence of a power law is raised.

JEL classification: D4, D7, and G0.

Key words Partition vector, Aggregate variability, jump Markov processes, Ewens distribution, Power-laws.
\end{abstract}

\footnotetext{
${ }^{1}$ A preliminary version was presented at the Workshop on Economics of Heterogenous Interacting Agents, Univ. Genoa, Italy, June, 1999. The research is supported in part by the UCLA Academic Senate grant. The author wishes to thank Richard H. Day, Thomas Lux and anonymous referee for their comments. I owe Domenico Costantini for the Zabell reference.
} 


\section{Introduction}

Behavior of macroeconomic variables in markets emerges as aggregate effects of, or determined by excess demand behavior of a large number of interacting economic agents. There have been a veritable explosion of empirical and simulation studies of financial markets, in particular, that are aimed at shedding light on behavior of market clearing prices, returns, or volumes of transactions. $^{2}$

Agents in the markets employ various strategies or trading rules. When we put into the same group or cluster all agents with the same strategy, trading rule, excess demand function, and the like, there typically will be many clusters in the markets. For convenience of reference, we identify agents with the rules they employ, and say that agents of the same type form a group or cluster.

Clusters evolve over time as agents switch their decision rules or behavioral patterns in response to changing economic environments, such as changing market sentiments and the like. It is impossible to say in advance how many clusters are going to be present at any given time. We can only sample some numbers of agents and count the number of different strategies being used at a particular time. There can be, in principle, infintely many potential strategies. For example, random combinations of two basic algorithms, say, in different proportions produce different strategies, because they will have different expected performance and varinces or risk characteristics. New decision or trading rules will be invented in the future, and so on. Knowing the types of agents present in a market is analogous, indeed formally identical, to the so-called "sampling of species problem" faced by statisticians concerned with the evolution of biological species, as for example described in Zabell (1992). The purpose of this paper is accordingly the application of mathematical theory in the latter field to the interactive agent problem in economics. I first describe a jump Markov process to examine the distribution of the sizes of clusters of agents by strategy types.

To analyze behavior of such markets, we consider order statistics of shares of types, that is, we derive distributions of the normalized sizes of the clusters in non-increasing order, and concentrate on the first several largest clusters or groupings of agents, if the size distributions are such that most probabilities are concentrated on the first few order statistics. Examining a few such large clusters will give us approximate behavior of markets as a

\footnotetext{
${ }^{2}$ See Gopikrishnan et al.,(1998), Lux and Marchesi (1999), Mantegna and Stanley (1994), and Takayasu and Sato (1997), among many. Reported simulation studies of financial markets by multi-agents models apparently mimic well some, but not all, aspects of the real world behavior of returns. See Lux and Marchesi (1999), for example. One feature of the real world financial markets which is not apparently adequately modeled in early simulation models is volatility. Volatility of the prices (returns) tends to decreases and becomes (too) small as the total number of agents is increased. (Private communication, Lux (1999).) One reason for this simulation finding seems to be the use of closed models in the simulations, that is, the total number of agents, $N$, is held fixed in conducting simulation, and two or more simulation results are compared with different but fixed $N$ values. To find a possible cause for this simulation finding, this paper employs an open model in which the total number of agents of all types is random by allowing entries and exits, in addition to changes of types of agents (changes in strategies employed by agents).
} 
whole, as we show later in this paper. This is especially true when agents are positively correlated in the sense we make precise later.

We are interested in situations in which a few of the large fractions of the types of agents, whatever the types may be, dominate, and approximately determine the market excess demand for the shares. The joint distribution for the largest $r$ fractions of the agents classified by their trading rules, is derived and used to characterize approximately the market excess demands, and the market clearing price movements. In the last part of the paper, $r$ is taken to be two. Our approach thus provides a stochastic generalization of a deterministic model of a share market such as Day and Huang (1990), ${ }^{3}$ by providing microscopic probabilistic process for agents changing their strategies. In this paper we discuss only jump Markov processes with exogenously specified transition rates. By suitably endogenizing them, our formulation allows for effects of social influences such as group sentiments or pressure, and herd-like behavior, that is, bandwagon effects, and so on by appropriate choices of transition rates for jump Markov processes. This is briefly suggested in Section 8. Also consult Aoki (2001) for several examples on how to model these effects as jump Markov processes.

\section{The Distribution of Agent Types}

In this and the next section two alternative characterizations of state of the market are described. This section employs the more familiar one of the two. It is called empirical distribution in the statistics literature. The other will be introduced in section 3 . Here we give an example of jump Markov processes which leads to the distribution of section 3 , in order to show that our framework of modeling agent behavior is rather natural in examining clucters formed by agents.

Suppose that there are potentially a large number $K$ of types of agents who participate in a market. For the moment suppose that the value of $K$ is known. Then, the vector $\mathbf{n}=\left(n_{1}, n_{2}, \ldots, n_{K}\right)$ describes how $n$ agents are distributed over $K$ types, $n=n_{1}+n_{2}+\cdots+n_{K}$. In this section we use this vector as state vector. Excluding pathological phenomena of infinitely many jumps in an infinitesimally small time interval, transition rates from one category to the others uniquely determine the corresponding jump Markov processes. See Breiman (1969) for example.

In our open model, we need to specify entry rates, exit rates and rates of type changes. The transition rate

$$
w\left(\mathbf{n}, \mathbf{n}+\mathbf{e}_{k}\right)=c_{k}\left(n_{k}+h_{k}\right),
$$

specifies entry rate to the market by agents of type $k$, for $n_{k} \geq 0$, where $\mathbf{e}_{k}$ is the vector with unit element in the $k$ th position and zero elsewhere. In this specification of the entry transition rate, the term, $c_{k} n_{k}$, stands for attractiveness of a large group, such as network externality which makes it easier for others to join the cluster or group. The other term, $c_{k} h_{k}$,

\footnotetext{
${ }^{3}$ Their model is deterministic and discrete-time with two types of agents of fixed number, namely one of each.
} 
represents new entry to the market which is independent of cluster size, and is similar to the immigration term in the literature of birth-and-death-with immigration models.

The second rate is that of the exit or departure from the market by type $j$ agents

$$
w\left(\mathbf{n}, \mathbf{n}-\mathbf{e}_{j}\right)=d_{j} n_{j}
$$

for $n_{j} \geq 1$, and the last specifies the transition rate of changing types by agents from type $j$ to type $k$, that is, switching of trading rules by agents

$$
w\left(\mathbf{n}, \mathbf{n}-\mathbf{e}_{j}+\mathbf{e}_{k}\right)=\lambda_{j k} d_{j} n_{j} c_{k}\left(n_{k}+h_{k}\right)
$$

with $\lambda_{j k}=\lambda_{k j}$, and where $j, k=1,2, \ldots K$. We assume that $d_{j} \geq c_{j}>0$, and $h_{j}>0$, and $\lambda_{j k}=\lambda_{k j}$ for all $j, k$ pairs.

This jump Markov process thus specified has the steady state or stationary distribution

$$
\pi(\mathbf{n})=\prod_{j=1}^{K} \pi_{j}\left(n_{j}\right)
$$

where

$$
\pi_{j}\left(n_{j}\right)=\left(1-g_{j}\right)^{-h_{j}}\left(\begin{array}{c}
-h_{j} \\
n_{j}
\end{array}\right)\left(-g_{j}\right)^{n_{j}}
$$

where $g_{j}=c_{j} / d_{j}$. These expressions are derived straightforwardly by applying the detailed balance conditions to the transition rates. See Kelly (1979, Chapt.1) for example. However, the model in this form ignores the possibility of correlation in agent behavior. ${ }^{4}$ To allow for that, we proceed to a method for deriving the size distribution of strategy clusters.

\section{The Size Distribution of Strategy Clusters}

Now, we introduce the second state vector, called the partition vector by Zabell (1992), $\mathbf{a}=\left(a_{1}, a_{2}, \ldots, a_{n}\right)$, where $a_{k}$ is the number of types or clusters with exctly $k$ agents. Consequently we have an inequality

$$
\sum_{i=1}^{n} a_{i}=K_{n} \leq K
$$

where $K_{n}$ is the number of groups or clusters formed by $n$ agents, and

$$
\sum_{i=1}^{n} i a_{i}=n
$$

which is an accounting identity. ${ }^{5}$

\footnotetext{
${ }^{4}$ Lack of correlation among agent behavior are assumed in other economic models. For example, Sutton (1992) noted this in connection with the Gibrat's law.

${ }^{5}$ The partition vector is originally introduced by Kingman under a different name. We use the name introduced by Zabell because it is appropriate in our applications. The set of agents is partitioned into subsets, each of which is composed of agents of the same type. What we call clusters in this paper correspond to the exchangeable random partitions in Kingman (1978a, b).
} 
To further simplify our presentation, let us suppose that $h_{j}=h$ and $g_{j}=g$ for all $j$. Because there are $a_{j}$ of the $n$ 's which equal $j$, it follows that

$$
\pi(\mathbf{n})=\left(\begin{array}{c}
-K h \\
n
\end{array}\right)^{-1} \prod_{j=1}^{K}\left(\begin{array}{c}
-h \\
j
\end{array}\right)^{a_{j}}
$$

Now let $K$ become very large to allow for the possibility of an indefinitely many number of types. To keep the mean finite we keep $h$ very small, while the product $K h$ approaches a positive constant $\theta$. We note that the negative binomial expression

$$
\left(\begin{array}{c}
-h \\
j
\end{array}\right)^{a_{j}}
$$

approaches $(h / j)^{a_{j}}(-1)^{j a_{j}}$ as $h$ becomes smaller. Suppose $K_{n}=k<K$. Then, there are

$$
\frac{K !}{a_{1} ! a_{2} ! \cdots a_{n} !(K-k) !}
$$

many ways of realizing a vector. Hence

$$
\pi(\mathbf{a})=\left(\begin{array}{c}
-\theta \\
n
\end{array}\right)(-1)^{n} \frac{K !}{a_{1} ! a_{2} ! \cdots a_{n} !(K-k) !} \prod_{j}\left(\frac{h}{j}\right)^{a_{j}} .
$$

Noting that $K ! /(K-k) ! \times h^{k}$ approaches $\theta^{k}$ in the limit of $K$ becoming infinite and $h$ approaching 0 while keeping $K h$ at $\theta$, we arrive at the probability distribution known as the Ewens distribution, or Ewens sampling formula ${ }^{6}$

$$
\pi_{n}(\mathbf{a})=\frac{n !}{\theta^{[n]}} \prod_{j=1}^{n}\left(\frac{\theta}{j}\right)^{a_{j}} \frac{1}{a_{j} !}
$$

where $\theta^{[n]}:=\theta(\theta+1) \cdots(\theta+n-1)$. This is called an ascending factorial of $\theta$. We next examine some of its properties following Watterson (1976).

\subsection{The number of clusters and value of $\theta$}

Ewens sampling formula has a single positive-valued parameter $\theta$. Its value influences the number of clusters formed by the agents. Smaller values of $\theta$ tends to produce a few large clusters, while larger values produce a large number of clusters of smaller sizes.

To obtain quickly some intuitive understanding of the effects of the value of $\theta$ on the cluster size distributions, take $n=2$ and $a_{2}=1$. All other $a$ s are zero. Then

$$
\pi_{2}\left(a_{1}=0, a_{2}=1\right)=\frac{1}{1+\theta} .
$$

\footnotetext{
${ }^{6}$ This distribution is very well known in the genetics literature, see Ewens (1972), Kingman (1978a, b), or Johnson, Kotz, and Balakrishnan (1997).

This distribution has been investigated by Arratia and Tavaré (1992), or Hoppe (1987) among several others. Kingman (1980) states that this distribution arise in many applications. There are other ways of deriving this distribution. See Costantini and Garibaldi (1999).
} 
This shows that two randomly chosen agents are of the same type with high probability when $\theta$ is small, and with small probability when $\theta$ is large.

Two extreme situations also reveal connections between the value of $\theta$ and the number of clusters. We note that the probability of $n$ agents forming a single cluster is given by

$$
\pi_{n}\left(a_{j}=0,1 \leq j \leq(n-1), a_{n}=1\right)=\frac{(n-1) !}{(\theta+1)(\theta+2) \cdots(\theta+n-1)}
$$

while the probability that $n$ agents form $n$ singletons is given by

$$
\pi_{n}\left(a_{1}=n, a_{j}=0, j \neq 1\right)=\frac{\theta^{n-1}}{(\theta+1)(\theta+2) \cdots(\theta+n-1)} .
$$

With $\theta$ much smaller than one, the former probability is approximately equal to 1 , while the latter is approximately equal to zero. When $\theta$ is much larger than $n$ the opposite is approximately true.

Recall the definition of the ascending factorials in (2). From (2), Hoppe (1987) has shown that the probability of $n$ agents forming $k$ clusters is given by

$$
\operatorname{Pr}\left(K_{n}=k\right)=\frac{1}{\theta^{[n]}} c(n, k) \theta^{k},
$$

where $c(n, k)$ are the polynomial coefficients ${ }^{7}$ in the exapnsion of $\theta^{[n]}$

$$
\theta^{[n]}=\sum_{1}^{n} c(n, k) \theta^{k}
$$

We can use this formula to verify that the expected number of types increase with $\theta$. As $\theta$ goes to infinity, the expected number of types approaches $n$, namely, total fragmentation of agents in the sample by types. For small values of $\theta$, Ewens has shown that the mean number of clusters formed by $n$ agents is

$$
E\left(K_{n}\right)=\sum_{j=0}^{n-1} \frac{\theta}{\theta+n-j} \approx 1+\theta[\ln (n-1)+\gamma],
$$

where $\gamma=.577$ is the Euler's constant.

\subsection{Fractions}

The expected value of $a_{j}$ is given by

$$
E\left(a_{j}\right)=\sum_{w(n)} a_{j} \pi_{n}(\mathbf{a})=\frac{\theta}{j} \frac{n !}{(n-j) !} \frac{\theta^{[n-j]}}{\theta^{[n]}},
$$

where $w(n):=\left\{\mathbf{a}: \sum_{j} j a_{j}=n\right\}$. A quick way to see this is to recognize that the multiplication of $\pi_{n}$ by $a_{j}$ is equivalent to changing the partition vector

\footnotetext{
${ }^{7}$ These are known as the signless Stirling numbers of the first kind. Stirling numbers are discussed in van Lint and Wilson (1992, p. 104) for example. This number is the number of permutations of $n$ symbols with exactly $k$ cycles. In Hoppe, his urn model of the Ewens distribution makes the occurence of this number natural.
} 
from a to $\mathbf{a}^{\prime}$ where the $j$ th component is changed from $a_{j}$ to $a_{j}-1$ and the sum $\sum i a_{i}=n$ is changed to $\sum i a_{i}^{\prime}=n-j$, because $a_{i}^{\prime}=a_{i}, i \neq j$, but $a_{j}^{\prime}=a_{j}-1$. We can evaluate the effects of increasing correlations or mutual dependence on the size of $E a_{j}$, by taking partial derivative of it with respect to $\theta$ : As $\theta$ increases, $E a_{j}$ for $j$ much smaller than $n$ increases linearly in $\theta$.

Watterson (1976) shows how to calculate the variance and covariances by using the relation

$$
E\left\{a_{j}\left(a_{j}-1\right)\right\}=\left(\frac{\theta}{j}\right)^{2} \frac{n !}{(n-2 j) !} \frac{\theta^{[n-2 j]}}{\theta^{[n]}},
$$

and for $i \neq j$

$$
E\left\{a_{i} a_{j}\right\}=\frac{\theta^{2}}{i j} \frac{n !}{(n-i-j) !} \frac{\theta^{[n-i-j]}}{\theta^{[n]}} .
$$

What is characteristic of this distribution is the fact that the standard deviations of $a$ s are of the same order of magnitude as the means.

Let $x_{i}=i a_{i} / n$. Since $a_{i}$ is, by definition, the number of clusters of size $i$, that is, the number of clustes, each formed by $i$ agents, this is the fraction of agents in clusters of size $i$. These fractions are in the infinite dimensional simplex, $\sum_{i} x_{i} \leq 1$. Its expected value is

$$
E\left(x_{j}\right)=E\left(j a_{j} / n\right) .
$$

The order statistics of the fractions, $x_{(1)} \geq x_{(2)} \geq \cdots$ are important in markets with highly correlated agents. With $\theta$ smaller than 1 , the sum of two or three largest fractions can be shown to be nearly one. See Table III of Watterson and Guess (1977) where numerical values of the expected values of the largest fraction is tabulated for different values of $\theta$. For example, with $\theta=.3, .4$, and .5 , the expected value of the largest fraction is $E\left(x_{(1)}\right)=.84, .79$, and .76 , respectively. They obtained these numbers numerically. We describe some theoretical background in the next section.

\section{The Largest Share}

\subsection{The Expected Value}

Here we follow Watterson (1976), and Watterson and Guess (1977) to sketch how the probability density function of the largest order statistics of $x$ 's, $x_{(1)}$, is derived. An entirely analogous procedure can calculate the joint probability density for $r$ order statistics of the fractions. This is discussed in the Appendix.

Start with a finite $K$. We can assume that $x_{K}$ is $x_{(1)}$, i.e., assume without loss of generality that

$$
0 \leq x_{i} \leq x_{K}=1-x_{1}-\cdots-x_{K-1}
$$

for $i=1, \ldots, K-1$. Change variables from $x$ s to

$$
y_{i}=x_{i} /\left(1-x_{1}-x_{2}-\cdots-x_{K-1}\right) \leq 1 \text {. }
$$


Then, we have

$$
E\left(x_{(1)}\right)=K \int \cdots \int p_{K} \phi\left(x_{1}, \cdots, x_{K}\right) d x_{1} d x_{2} \cdots d x_{K-1},
$$

where $\phi$ is the symmetric Dirichlet distribution with parameter $\epsilon$, that is

$$
\phi\left(x_{1}, x_{2}, \ldots, x_{K}\right)=\frac{\Gamma(K \epsilon)}{\left(\Gamma(\epsilon)^{K}\right.} \prod_{i=1}^{K} x_{i}^{\epsilon-1}
$$

is the joint density function of $x \mathrm{~s}^{8}$

Noting that $1-x_{1}-\cdots-x_{K-1}=\left(1+\sum_{j=1}^{K-1} y_{j}\right)^{-1}$, and that the Jacobian is

$$
\frac{\partial \mathbf{x}}{\partial \mathbf{y}}=\left(1+\sum_{j} y_{j}\right)^{-K}
$$

by straightforward calculation, we rewrite

$$
E\left(x_{(1)}\right)=\frac{K \Gamma(K \epsilon)}{(\Gamma(\epsilon))^{K}} \int \cdots \int \prod_{j=1}^{K-1} y_{j}^{\epsilon-1}\left(1+\sum_{j} y_{j}\right)^{-1-K \epsilon} d y_{1} \cdots d y_{K-1}
$$

where the range of integration is over the cube, $0 \leq y_{j} \leq 1, j=1, \ldots, K-1$. By multiplying the numerator and denominator by $\epsilon^{K-1}$, we regard this integral as the expected value of $\left(1+Z_{K-1}\right)^{-1-K \epsilon}$ with respect to $Y_{j}, j=$ $1, \ldots, K-1$ which are independent and identically distributed with the density $\epsilon^{K-1} \prod_{j=1}^{K-1} y_{j}^{\epsilon-1}$.

Although we cannot express the expected value in closed form we can give the Laplace transform of $Z_{K-1}$ and obtain its limit as $K$ goes to infinity. From independence of $Y \mathrm{~s}$

$$
E\left(e^{-s Z_{K-1}}\right)=\left\{E\left(e^{-s Y_{1}}\right)\right\}^{K-1}=\left\{\int_{0}^{1} e^{-s y} \epsilon y^{\epsilon-1} d y\right\}^{K-1}
$$

Write the integrand as

$$
\epsilon e^{-s y} y^{\epsilon-1}=\epsilon\left\{y^{\epsilon-1}+\left(e^{-s y}-1\right) y^{\epsilon-1}\right\}
$$

and let $K$ go to infinity, and $\epsilon$ to zero, while the product goes to a nonnegative value $\theta$. Thus

$$
E\left(e^{-s Z_{K-1}}\right) \rightarrow \exp \left\{\theta \int_{0}^{1}\left(e^{-s y}-1\right) y^{-1} d y\right\}
$$

Denote by $Z$ the limiting random variable with the Laplace transform specified above. Then

$$
E\left(x_{(1)}\right)=e^{\gamma} \theta \Gamma(\theta+1) E\left\{(1+Z)^{-1-\theta}\right\} .
$$

\footnotetext{
${ }^{8}$ The appearance of Dirichlet distributions here may seem arbitrary. Actually there is a deep mathematical relation between the exchangeable random partitions introduced by Kingman (1978a, b) and its representation using the Dirichlet measures, see Zabell (1992). We do not stop here to explain these but go directly to calculate the expected size of the largest fraction goverend by the Dirichlet distribution. Also see Kingman (1993).
} 
In deriving this expression we note that $\Gamma(K \epsilon) \rightarrow \Gamma(\theta), \Gamma(\epsilon)=\Gamma(1+\epsilon) / \epsilon$, and that $\Gamma(1+\epsilon) \approx \Gamma(1)+\Gamma^{\prime}(1) \epsilon=1-\gamma \epsilon$, where $\gamma=-\Gamma^{\prime}(1)$ is Euler's constant. See Abramovitz and Stegun [(1968, (5.1.1) and (5.1.11)]. By definition

$$
\Gamma(1+\theta)=\int_{0}^{\infty} x^{\theta} e^{-x} d x
$$

and we note that

$$
\Gamma(\theta+1)(1+Z)^{-1-\theta}=\int_{0}^{\infty} s^{\theta} e^{-(1+Z) s} d s
$$

Taking expectation with respect to $Z$, we obtain

$$
E\left(x_{(1)}\right)=e^{\gamma \theta} \int_{0}^{\infty} s^{\theta} e^{-s} E\left(e^{-s Z}\right) d s=\int_{0}^{\infty} e^{-s} e^{-\theta E_{1}(s)} d s
$$

where we use the relation

$$
E\left(e^{-s Z}\right)=\exp \left\{-\gamma \theta-\theta \ln t-\theta E_{1}(s)\right\}
$$

and where

$E_{1}(s)=\int_{s}^{\infty} e^{-x} x^{-1} d x=-\gamma-\ln s-\sum_{n=1}^{\infty} \frac{(-s)^{n}}{n n !}=-\gamma-\ln s-\int_{0}^{\infty}\left(e^{-s y}-1\right) d y$.

The marginal probabilty density of the largest fraction is

$$
f(x)=\theta x^{-1}(1-x)^{\theta-1}, \quad 1 / 2<x \leq 1 .
$$

When $x$ is not greater than $1 / 2$, the expression is more complex

$$
f(x)=\Gamma(\theta+1) e^{\gamma \theta} x^{\theta-2} g\left(x^{-1}-1\right), \quad 0 \leq x \leq 1 / 2,
$$

where $g(\cdot)$ is the density of the random variable $Z$ introduced above, and characterized in terms of its Laplace transform.

See Appendix for the joint probability density for the first $r$ largest fractions. The expression for $r=2$ is used in Sections 5 and 6 .

\subsection{The most probable values}

Let $x$ and $y$ be the two largest fractions. Their joint density is

$$
f(x, y)=\theta^{2}(x y)^{-1}(1-x-y)^{-1 / 2},
$$

in the region $x+2 y \geq 1$, and $x+y \leq 1$. Its partial derivative with respect to $y$ vanishes on the line $2 x+3 y=2$, which is located in the region where the expression given above holds. This line is a ridge along which the most probable values of $y$, given $x$ are located. With $\theta=.5, E(x)=.758$ by Table III of Watterson and Guess. Approximating $y$ by the most probable value, $y \approx 2 / 3-2 x / 3$, we calculate $E y$. We also know that $E y \approx E x \theta(\ln 2-\theta / 2)$. They both give value $E y \approx .16$. We may approximate $y$ by the equation for the most probable $y$ without too much error. 


\section{Market Volatility}

Here we show that our model has non-vanishing volatility as the number of participants goes to infinity unlike some simulation models with exogenously fixed numbers of agents.

Let $E(x)$ and $E(y)$ be the expected values of the two laragest fractions, $x$ and $y$. Watterson (1976) shows that

$$
E(y) \geq \theta E(x) B_{1 / 2}(0, \theta+1) \approx \theta E(x)(\ln 2-\theta / 2),
$$

where $B_{1 / 2}$ is an incomplete beta function, see Abramovitz and Stegun $(1968,26.5)$. Using this formula, $E(y) \approx .16$ with $\theta=.4$, hence $E(x)+$ $E(y) \approx .95$. Similarly, we have $E(x+y)=.97$,, and .92 for $\theta=.3$, and .5 , respectively. We may therefore think of $\theta$ about 0.4 . With $\theta=.4$, the expected numbers of clusters are $E\left(K_{10}\right)=2.1, E\left(K_{100}\right)=3.0, E\left(K_{1000}\right)=4.0$, $E\left(K_{10^{5}}\right)=5.8$, and $E\left(K_{10^{7}}\right)=7.7$. These figures indicate that there are several small fractions in addition to the two large ones when the number of participants are $n \geq 100$.

Watterson also has bounds for other moments with $k$ and $l$ non-negative integers

$$
E\left(x^{k} y^{l}\right) \geq G \theta^{2} \Gamma(\theta) e^{\gamma \theta} B_{1 / 2}(k, l+\theta),
$$

with $G=E(x) / \theta \Gamma(\theta) e^{\gamma \theta}$, and where $B_{1 / 2}(a, b)$ is the incomplete beta function. The inequality comes from approximation he used to evaluate some integrals. Abramovitz and Stegun have some series expansions for the incomplete beta functions. Unfortunately the bounds are not sharp enough to give precise bounds on the variances of $x$. If we use $y \approx .95-x$, then

$$
E(x y) / E(x)=\theta B_{1 / 2}(1,1+\theta) \approx[\theta /(1+\theta)]\left[1-\left(\frac{1}{2}\right)^{1+\theta}\right],
$$

may be used to estimate

$$
\sqrt{\operatorname{var}(x)} / E(x) \approx .21
$$

In other words, the standard deviation of the largest fraction is about $1 / 5$ of its mean. See Watterson (1976) and Watterson and Guess (1977) for more precise calculation procedures.

What is most remarkable when $n$ is large is that some small numbers of configurations account for majority of possible patterns. ${ }^{9}$ That is, some small number of configurations are most likely to be realized or observed. This justifies studying markets with a small number of strategy clusters.

\section{Market Excess Demand Behavior}

In this section we derive approximate expression for the market excess demands with two large fractions $x$ and $y$, which approximately sum to 1 . In the previous section, we have shown that about 95 per cent of the total

\footnotetext{
${ }^{9}$ This feature has been noticed in other contexts as well. Mekjian (1991) compares genetic and physics examples.
} 
market participants belong to the two largest subgroups of agents by types. With two largest clusters, there are two regimes; one with a cluster of agents with strategy 1 is the largest share, and the other with a cluster of agents using strategy 2 being the largest. For ease of comparison, we use the same individual excess demand function forms as in Day and Huang. The agents of strategy 1 has the excess demand

$$
d_{1}(P)=(u-P) h(P),
$$

and agents with strategy 2 has the excess demand

$$
d_{2}(P)=-(u-P),
$$

with $h(P)=[(P-m)(M-P)]^{-1 / 2}$ where we have set $a=b=1$ in their specification and set $u=(M+m) / 2$ without loss of generality. In the language of Day and Huang, agents with strategy 1 are the fundamentalists and those with strategy 2 are chartists. We note that the two excess demands are of opposite sign, i.e., the two types of agents are on the opposite side of the market.

Let $P$ denote the price of the shares, and let $d_{x}(P)$ denote individual excess demand of the type which happens to be the largest fraction $x$. Similarly for $d_{y}(P)$.

\subsection{Zero Excess Demand}

The market excess demand, $D$, is then given by summing over individual excess demands

$$
D(P) / n=x d_{x}(P)+y d_{y}(P) .
$$

Set the right-hand side to zero to define the critical prices at which the zero market excess demand is realized. In the case where the agents in the largest cluster are using strategy 1 , there are three prices at which the market excess demand is zero if the inequality $(M-m) / 2 \geq(x / y)$ holds. One is $P=u$, and the other two are given by the roots of

$$
h(P)=y / x,
$$

or $P^{2}-2 u P+M m=(x / y)^{2}$. Denote them by $P^{*}$, and $P_{*}$, where $P_{*}<u<$ $P^{*}$. These critical values depend on $x$ and $y$, as shown below.

If $(M-m) / 2 \leq x / y$, then $P=u$ is the only price which produces the zero excess demand. To be definite we assume that $(M-m) / 2$ is sufficiently large for the three critical prices to exist. ${ }^{10}$ In the other regime where the agents in the largest cluster are using strategy 2 there are also three critical points if the condition holds with $y / x$ replacing $x / y$. We proceed to examine the case with three critical prices in both regimes.

Noting that $P^{*}=u+\sqrt{(M-m)^{2} / 4-(x / y)^{2}}$ in the first regime and $P^{*}=u+\sqrt{(M-m)^{2} / 4-(y / x)^{2}}$ in the second regime, the two critical prices $P^{*}$ and $P_{*}$ are further apart in regime 2 than in regime $1 ; m<$

\footnotetext{
${ }^{10}$ If $y / x \leq(M-m) / 2 \leq x / y$, then regime 1 has a unique $P=u$, but in regime 2 there are three critical points.
} 
$P_{*}(2)<P_{*}(1)<u<P^{*}(1)<P^{*}(2)<M$, where we use the notation such that $P^{*}(2)$ denotes the largest critical price under regime 2 , and so on.

In Section 7 we provide approximate analysis of price behavior using a difference equation because of mathematical intractabililty of dealing with continous time stochastic differential equations. The derivative of the market excess demand with respect to $P$ is

$$
D^{\prime}(P)=y-\left(\frac{M-m}{2}\right)^{2} h(P)^{3} x
$$

in regime one and in particular

$$
D^{\prime}(u)=-f(u) x+y=-\frac{2}{M-m} x+y,
$$

in regime 1 , which is positive, and less than 1 , when $M-m$ is sufficiently large, that is, the critical price $P=u$ is locally stable.

The difference equation are locally unstable at the other two critical prices at which the market excess demand vanish because

$$
D^{\prime}\left(P^{*}\right)=-\left(u-P^{*}\right)^{2} h\left(P^{*}\right)^{3}<-1,
$$

and

$$
D^{\prime}\left(P_{*}\right)=-\left(u-P_{*}\right)^{2} h\left(P_{*}\right)^{3}<-1,
$$

again for sufficiently large $M-m$.

\subsection{Volatility of Market Excess Demand}

In Sec. 4.1 we have exhibited the expression for the probability density of the largest fraction $x$. The expression for the two largest fraction is

$$
g(x, y)=\theta^{2} x^{-1} y^{-1}(1-x-y)^{\theta-1}
$$

for $0 \leq y \leq x \leq 1$, and $x+2 y \geq 1$.

In regions of $x<1 / 2$ and $x+2 y<1$ more complicated formula obtains and we must resort to numerical determination of the density. With $\theta$ small, it is likely that both the inequalities $x>1 / 2$ and $x+2 y \geq 1$ hold. We proceed on this assumption in evaluating the expected values.

The upper and lower market clearing prices are functions of the fractions $x$ and $y$. To investigate the effects of the fractions deviating from their means on the critical prices, let $\delta P^{*}=P^{*}-P^{*}(E x, E y)$ denote the deviation of the upper critical price from that when the fractions are at their mean values, where dependence of $P^{*}$ on $x$ and $y$ is not explicitly shown.

We have

$$
\delta P^{*}=-B\left(\frac{\delta x}{E x}-\frac{\delta y}{E y}\right),
$$

where $B:=(E x / E y)^{2} /(\bar{P}-u)$ with $\bar{P}=P^{*}(E x, E y)$. is a constant. This is the expression in regime 1 . In regime 2 , the roles of $x$ and $y$ are interchanged. The expression for $\delta P_{*}$ in regime 2 is the negative of this.

From this we obtain the variance of $\delta P^{*}$ in terms of variances and covariance of $x$ and $y$. We note only the coefficient $B$ vary by changes of regimes. 
Proposition: Volatilities of prices near the upper and lower critical values are greater under regime 1 than regime 2 , if $(M-m)^{2} / 4>\left[(E x / E y)^{2}+\right.$ $\left.(E y / E x)^{2}\right]^{-1}$.

\section{Approximate Dynamics for Price Differences and Power Law}

In this section we discuss behavior of large price changes, and returns, in heuristic terms. We fix time interval $\Delta$ and write the recurrent equation for share price as

$$
P_{t+\Delta}=P_{t}+\kappa D\left(P_{t}, \xi_{t}\right)
$$

where $\kappa=c \times \Delta$ is an adjustment constant, and $\xi_{t}$ stands for the twodimensional vector with components $x_{t}$ and $y_{t}$.

Let $\rho_{t}$ be the price difference $P_{t}-P_{t-\Delta}$ for some small $\Delta$.

Then, the price difference is governed by the difference equation

$$
\rho_{t+\Delta}=A_{t} \rho_{t}+B_{t}
$$

with $A_{t}=1+\kappa D^{\prime}\left(P_{t-\Delta}, \xi_{t-\Delta}\right)$, where prime indicates partial derivative with respect to $P$, and $B_{t}=\kappa D_{\xi}\left(P_{t-\Delta}, \xi_{t-\Delta}\right)\left(\xi_{t}-\xi_{t-\Delta}\right)$, where the subscript $\xi$ indicate the gradient vector with respect to $x$ and $y$.

In the mathematical literature we do not find theory to examine behavior of this difference equation, since the existing theory requires that $\left(A_{t}, B_{t}+d_{n}(t)\right)$ be i.i.d., see Kesten (1973), Vervaat (1979), Letac (1986), Goldie (1991), or de Haan et al.(1989). In particular, the condition of identically distributed pair of random variables may not be valid in general. However, the condition approximately holds in the neighborhood of $P=u$, or $P_{*}$ or $P^{*}$. Alternatively, we may appeal to Brandt (1986), who derives the conditions under the assumption of ergodic stationarity for $\left(A_{t}, B_{t}\right)$, rather than the i.i.d. conditions used by other writers.

We assume that under certain technical conditons, a stationary distribution exists for $\rho_{\infty}=A_{1} \rho_{\infty}+B_{1}$. See also Sornette (1998), or Takayasu and Sato (1997), among others. We know that the distribution of the price differences will have a stationary statistical distribution with power law, that is the expression

$$
\operatorname{Pr}\left(\left|\rho_{\infty}\right|>z\right)=c z^{-\gamma}
$$

holds with some constants $c$, and $\gamma$ under some technical conditions. In particular the index $\gamma$ is determined by

$$
E\left(A_{1}^{\gamma}\right)=1 \text {. }
$$

This condition, if satisfied, will determine $\gamma$ by

$$
\gamma=1+\frac{2}{\kappa} \frac{E\left(-D^{\prime}\right)}{E\left(D^{\prime 2}\right)}
$$

where

$$
\frac{E\left(-D^{\prime}\right)}{E\left(D^{\prime 2}\right)}=\frac{\Theta E(x)-E(y)}{\Theta^{2} E\left(x^{2}\right)-2 E(x y) \Theta+E\left(y^{2}\right)} \approx \frac{\Theta-.18}{.18(\Theta+2 / 3)^{2}-(4 / 3) \Theta-.35},
$$


with $\Theta=\left(\frac{2}{M-m}\right)^{2} h\left(\rho_{\infty}\right)^{3}$. Then, with a large $\Theta$ we have an approximate expression $\gamma \approx 1+10 / \kappa \Theta$. Recall that $\kappa$ measures the adjustment speed of the price dynamics in reducing the magnitude of excess demands. Faster adjustment, other things being equal, will give smaller $\gamma$ values.

Returns may be similarly analyzed.

\section{Concluding Discussion}

This paper uses open models formalized as jump Markov processes to theoretically examine multi-agent models of financial markets. The paper then examines the market behavior by focussing on the distribution of the sizes of clusters of agents by strategy types. When agent behavior are positively correlated, that is, when the parameter $\theta$ in the Ewens distribution takes on a small positive value, we have shown that two largest clusters are likely to develop to which most agents belong. This provides some justification for studying economic models composed of many interacting agents of two or three strategy types.

By endogenizing some of the transition rates, social influences can be incorporated in the analysis of interacting agents by jump Markov processes. Aoki and Shirai (2000) does this in a context of search models. Aoki (2001) has other examples of incorporating what he calls as fields (aggregate) effects.

\section{References}

Abramovitz,M., and I. A. Stegun., Handbook of Mathematical Functions, Dover Ppublications, Inc., New York, 1968

Aoki, M., 1996, New Approaches to Macroeconomic Modeling: Evolutionary Stochastic Dynamics, Multiple Equilibria, and Externalities as Field Effects (Cambridge University Press, New York).

_ 1996a, "Shares in emergent markets" in Statistical Methods in Control and Signal Processes, T. Katayama, and S. Sugimoto (eds), Marcel Dekker, New York

_, 1998, "A Stochastic Model of Prices and Volumes in a Share Market with Two Types of Participants", Mimeo, Department of Economics, Univ. California, Los Angeles, presented at 1998 annual meeting, Soc. Econ.Dynamics, June, Philadelphia.

$\longrightarrow$, 2001, Modeling Aggregate Behavior and Fluctuations in Economics: Stochastic Views of Interacting Agents, forthcoming. Cambridge University Press, New York.

- and Y. Shirai, (2000), "A New Look at the Diamond Search Model: Stochastic Cycles and Equilibrium Selection in Search equilibrium", Macroeconomic Dynamics 4, 487-505.

Arratia, R., A. D. Barbour, and S. Tavaré. 1992 "Poisson process approximations for the Ewens sampling formula", Ann. Appl. Probab. 2, 519-35. 
Brandt, A., 1986, "The stochastic equation $Y_{n+1}=A_{n} Y_{n}+B_{n}$ with stationary coefficients,", Adv. Appl. Prob. 18, 211-20.

Breiman, L., Probability and Stochastic Processes: With a View Toward Applications, Houghton Mifflin, 1969, New York.

Chamayou, J-F., and G. Letac, 1991, "Explicit stationary distributions for

Costantini, D., and U. Garibaldi, 1999, 1998. "A Finitary Characterization of the Ewens Sampling Formula", mimeo, Univ. Bologna,

Day, R., and W. Huang, 1990, "Bulls, bears and market sheep", J. Econ. Behavior Organization 14, 299 -330.

de Haan, L, S. I. Resnick, , H. Rootzén, and C. de Vries, 1989, "Exremal behavior of solutions to a stochastic difference equation with applications to arch processes" Stoch. Proc. Appli. 32, 213-24.

Ewens, W. J., 1972, "The Sampling Theory of Selectively Neutral Alleles", Theor. Pop. Biol. 3, 87-112.

Goldie, C. M., 1991, " Implicit renewal theory and tails of solutions of random equations", Ann. Appl. Probab. 1, 126-66.

Gopikrishnan, M., M. Meyer, L. A. N. Amaral, and H. E. Stanley, 1998. "Inverse cubic law for the distribution of stock price variations" European Physical Journal B 3, 139-40.

Hoppe, F. M. 1984. "Polya-like urns and the Ewen's sampling formula", J. Math. Biology, 20, 91-4.

,- 1987 . "The sampling theory of neutral alleles and an urn model in population genetics", J. Math. Biology 25, 123-59.

Johnson, N., S. Kotz, and A. Balakrishnan,1997.Multivariate Discrete Distributions Wiley, New York.

Kelly, F., 1979. Reversibility and Stochastic Networks Wiley \& sons, New York.

1983 "Invariant measures and the Q-matrix ", in J. F. C. Kingman and G. E. H. Reuter (eds) Probability, Statistics, and Analysis, Cambridge Univ. Press, Cambrdige.

,- 1976 "On stochastic population models in genetics", J. Appl. Probab. 13,127-31.

Kendall, D. G.,1975. "Some problems in mathematical genealogy", in J. Gani (ed) Perspective in Probability and Statistics, Academic Press, New York.

Kesten, H.,1973. " Random difference equations and renewal theory for products of random matrices", Acta Math 131 208-48.

Kingman, J. F. K.,1969. "Markov population processes",J. Appl. Prob. 6, 1-18.

-1978a. "Random Partitions in Population Genetics", Proc. Roy. Soc. 361, 1-20.

-1978b. "The Representation of Partition Structure", J. Lond. Math. Soc.18, 347-80.

- 1980. Mathematics of genetic diversity SIAM, Philadelpha.

$\longrightarrow$, 1993.Poisson Processes Clarendon Press, Oxford

Lux, Thomas, and M. Marchesi, 1999. "Scaling and criticality in a stochastic multi-agent model of a financial market" Nature 397, 498-500. 
Mantegna, R. and H. E. Stanley, 1994, "Stochastic process with ultraslow convergence to a gaussian: the truncated Lévy flight" Phy. Rev. Lett $732946-49$.

- , and _ 1995, "Scaling behavior in the dynamics of an economic index", Nature 376 46-9.

Mekjian, A. Z. 1991. "Cluster distributuions in physics snd genetic diversity", Phy. Review A 44 8361-74.

Sornette, D. 1998. "Multiplicative processes and power laws", Phy.Rev. E57,4811-13.

Sutton, J.," Gibrat's Legacy",J. Econ. Literature, 35,40-59, 1997.

Takayasu, H., and A. Sato, 1997. "Stable infinite variance fluctuations in randomly amplified Langevin systems", Phy. Rev. Lett. 79, 966-697.

Tavaré, S., A. D. Barbour, and R. Arratia. 1997. "Approximating random combinatorial structures", Mimeo Univ. Southern Calif., Dept. Math.

van Lint, J. H., and R. M. Wilson. 1992. A Course in Combinatoric, , Cambdridge Univ. Press, Cambridge.

Vervaat, W., 1979."On a stochastic difference equation and a representation of non-negative infinitely divisible random variables", Adv. Appl. Prob. 11,750-83.

Watterson, G., A. 1976. "The stationary distribution of the infinitelymany neutral alleles diffusion model" J. Appl. Probab. 13, 639-51.

,- 1974 . "Models for the Logarithmic Species Abundance Distributions", Tho. Pop.Bio. 6, 217-50.

-, and H. A. Guess. 1977. "Is the most frequent allele the oldest?" Theor. Pop. Biol. 11 141-160.

Zabell, S. 1992. "Predicting the Unpredictable",Synthese 90,205-32.

\section{Appendix}

\subsection{Joint probability density for $r$ largest fractions}

We next derive the joint probability density for the largest $r$ fractions on the $K$-dimensional simplex, $x_{(1)} \geq x_{(2)} \geq \cdots \geq x_{(r)}$, where $x_{i}, i=1,2, \ldots, K$ are the fractions. Denote the Dirichlet probability density on the $K$ dimensional simplex by $\phi\left(x_{1}, x_{2}, \ldots, x_{k}\right)=\mathcal{D}(\epsilon, K)$. Then, the probability density for the first $r$ order statistics is given by

$f\left(x_{1}, x_{2}, \ldots, x_{r}\right)=K(K-1)(K-2) \cdots(K-r+1) \int \phi\left(x_{1}, x_{2}, \ldots, x_{k}\right) d x_{r+1} \cdots d x_{K-1}$,

where $1 \geq x_{1} \geq x_{2} \cdots \geq x_{r}>0$, and where we subsitute $x_{K}=1-x_{1}-\cdots-$

$x_{K-1}$. Carrying out the integral

$$
f\left(x_{1}, \ldots, x_{r}\right)=\frac{K !}{(K-r) !} \frac{\Gamma(K \epsilon}{\Gamma(\epsilon)^{K}}\left(x_{1} \cdots x_{r}\right)^{\epsilon-1} x_{R}^{(K-r) \epsilon-1} I
$$

where

$$
I=\int \cdots \int \prod_{r+1}^{K-1} y_{j}^{\epsilon-1}\left[1-a-x_{r}\left(y_{r+1}+\cdots+y_{K-1}\right)\right]^{\epsilon-1} d y_{r-1} \cdots d y_{K-1},
$$


where the integration is carried out in area $A$

$A=\left\{0 \leq y_{j} \leq 1, r+1 \leq j \leq K-1 ;\left(1-a-x_{r}\right) / x_{r} \leq y_{r+1}+\cdots+y_{K-1} \leq(1-a) / x_{r}\right\}$

where $a:=x_{1}+x_{2}+\cdots+x_{r}$.

As in the case of the largest fraction, introduce random variable $Z$ with the density function $g_{K-r-1}$ which is the $(K-r-1)$-fold convolution of the density $\epsilon y_{j}^{\epsilon-1}, j=r+1, \ldots, K-1$.

The integral is approximately given by

$$
I=\frac{\epsilon^{-(K-r)}}{x_{r}} g_{K-r-1}\left(\frac{1-a}{x_{r}}\right) .
$$

Letting $K \epsilon$ approach $\theta$, while $K$ goes to infinity and $\epsilon$ to zero, we note that $K(K-1) \cdots(K-r+1)$ approaches $K^{r}$, and

$$
\Gamma(\epsilon)^{K}=\left[\frac{\Gamma(1+\epsilon)}{\epsilon}\right]^{K}
$$

which approaches $\epsilon^{-K} e^{-\gamma \theta}$, where we use the fact

$$
\Gamma(1+\epsilon) \approx 1-\gamma \epsilon,
$$

where $\gamma$ is the Euler's constant, $\gamma=.5772 \cdots$.

Putting them all together we arrive at

$$
f\left(x_{1}, x_{2}, \ldots, x_{r}\right)=\theta^{r} e^{\gamma \theta} \Gamma(\theta) x_{r}^{\theta-1} g\left(\frac{1-a}{x_{r}}\right)\left(x_{1} x_{2} \cdots x_{r}\right)^{-1},
$$

in the range $1 \leq x_{1} \leq \cdots \leq x_{r}>0$, and $\sum_{1}^{r} x_{i} \leq 1$.

We know from our result for the largest fraction that $f\left(x_{1}\right)=\theta x_{1}^{-1}(1-$ $\left.x_{1}\right)^{\theta-1}$ for $x_{1}$ between $1 / 2$ and 1 , that is

$$
\Gamma(1+\theta) e^{\gamma \theta} g\left(\frac{1-x_{1}}{x_{1}}\right)=\theta\left(\frac{1-x_{1}}{x_{1}}\right)^{\theta-1},
$$

for $x_{1}$ between $1 / 2$ and 1 .

To obtain the expression for the density in the range $0 \leq x \leq 1 / 2$, we follow Watterson (1976) and differentiate the Laplace transform for the random variable $Z$

$$
E\left(e^{-s Z}\right)=\exp \left[\theta \int_{0}^{1}\left(e^{-s z}-1\right) z^{-1} d z\right]
$$

, with respect to $s$. Recall that this transform is derived in connection with the largest fraction. Then, divide the result by $-\theta$ to see

$$
(1 / \theta) \int_{0}^{\infty} e^{-s z} z g_{\theta}(z) d z=\int_{0}^{\infty} I_{(0,1]}(y) e^{-s y} d y \int_{0}^{\infty} e^{-s z} g_{\theta}(z) d z
$$

The right hand side is the product of two Laplace transforms. Hence the integrand of the left-hand side is the convolution of the uniform function on the unit interval and $g_{\theta}$

$$
(1 / \theta) z g_{\theta}(z)=\int_{z-1}^{z} g_{\theta}(y) d y
$$


Setting $z$ to 1 , we obtain

$$
g_{\theta}(1)=e^{-\gamma \theta} / \Gamma(\theta) .
$$

Differentiating the integral equation with respect to $z$, we derive the differential equation which determines the function recursively

$$
z g_{\theta}^{\prime}(z)+(1-\theta) g_{\theta}(z)=-\theta g_{\theta}(z-1)
$$

where $z \geq 0$. In the range $z \in[0,1)$, this integro-differential equation yields the result we obtained above. In the next range $z \in[1,2)$ we have

$$
g_{\theta}(z)=z^{\theta-1}\left[g_{\theta}(1)-\theta \int_{1}^{z} g_{\theta}(u-1) u^{-\theta} d u\right] .
$$

Changing variable of integration to $v=1 / u$, we note that the integration above becomes

$$
\int_{1 / z}^{1} v^{-1}(1-v)^{\theta-1} d v
$$

The joint density for the first two largest fractions is given by

$$
f_{\theta}^{(2)}(x, y)=\frac{e^{\gamma \theta} \theta^{2} \Gamma(\theta) y^{\theta-1}}{x y} g_{\theta}\left(\frac{1-x-y}{y}\right)=\frac{\theta^{2}}{x y}(1-x-y)^{\theta-1} .
$$

This expression is valid for the range $0<y<x<1,0<x+y<1$, and $x+2 y>1$, that is $y>(1-x) / 2$.

We know that

$$
g(z)=\frac{z^{\theta-1}}{\Gamma(\theta) e^{\gamma \theta}}
$$

for $z$ between 0 and 1. For other values of $z$, we have a recursion

$$
\frac{z g(z)}{\theta}=\int_{z-1}^{z} g(y) d y
$$

see Watterson and Guess (1977). Alternatively put, we have

$$
g(z)=z^{\theta-1}\left[g(n) n^{1-\theta}-\theta \int_{n}^{z} g(y-1) y^{-\theta} d y\right]
$$

in the range $n \leq z<n+1$. This can be verified by direct subsitution into the differential equation for $g_{\theta}$.

With the joint probability densities for the largest 2 or 3 fractions in the case of a large number of correlated agents in a market, we are in a position to approximately obtain the macroeconomic behavior of the market prices and quantieis. 